\title{
Metodologías de optimización del mantenimiento enfocado a transformadores de potencia: Una revisión del estado del arte
}

\author{
Preventive maintenance optimization methodologies focused on power \\ transformers: A state of the art review
}

\begin{abstract}
Sergio Raúl Villacrés-Parra. ${ }^{1}$, Mayra Alexandra Viscaíno-Cuzco. ${ }^{2}$ \& César Marcelo Gallegos-Londoño. ${ }^{3}$
\end{abstract}

Recibido: 24-06-2021 / Revisado: 01-07-2021 /Aceptado: 21-07-2021/ Publicado: 05-08-2021

\begin{abstract}
.
DOI: https://doi.org/10.33262/concienciadigital.v4i3.1.1827

Introduction. Of all the equipment that makes up the electrical system, the power transformer is one of the most important equipment due to its criticality, high maintenance costs, long repair times and the impact caused by a failure, both in the reduction of the reliability of the system, as in the losses generated to users; lead to avoiding the occurrence of failures is a matter of vital importance, for this maintenance plays a fundamental role. Aim. Identify the maintenance optimization algorithms that are applicable to power transformers, through a study of the state of the art. Methodology. It consisted of a systematic review of literature published in databases such as Scopus, carried out in three general stages: search, selection and analysis. Results. As a result of the search stage, 40 articles were obtained, finally 11 articles went to the analysis process; where it was identified that the most used optimization algorithms were: the one based on the Monte Carlo Simulation (27\%) and Linear Programming (18\%). 64\% of the investigations start the optimization process, on the implementation of a maintenance strategy, be it preventive and / or corrective. Conclusion. Optimization processes seek to

1 Escuela Superior Politécnica de Chimborazo, Facultad de Mecánica. Riobamba, Ecuador. sergio.villacres@espoch.edu.ec. https://orcid.org/0000-0002-9497-9795

2 En libre ejercicio profesional. Macas, Ecuador. mayraviscaino@hotmail.com. https://orcid.org/00000003-4987-7797

3 Escuela Superior Politécnica de Chimborazo, Facultad de Mecánica. Riobamba, Ecuador. cesar.gallegos@ espoch.edu.ec. https://orcid.org/0000-0002-8685-7501
\end{abstract}


minimize maintenance costs, maximize reliability, minimize asset deterioration, or a combination of them.

Keywords: Power transformer, maintenance optimization, optimization algorithm, maintenance cost, reliability.

\section{Resumen.}

Introducción. De todos los equipos que conforman el sistema eléctrico, el transformador de potencia es uno de los equipos más importantes debido a su criticidad, los elevados costos de mantenimiento, los prolongados tiempos de reparación y el impacto que causa un fallo, tanto en la reducción de la fiabilidad del sistema, como en las pérdidas generadas a los usuarios; conducen a que evitar la ocurrencia de los fallos sea un tema de vital importancia, para ello el mantenimiento desempeña un rol fundamental. Objetivo. Identificar los algoritmos de optimización del mantenimiento que sean aplicables a transformadores de potencia, a través de un estudio del estado del arte. Metodología. Consistió en una revisión sistemática de literatura publicada en bases de datos como Scopus, desarrollándose en tres etapas generales: búsqueda, selección y análisis. Resultados. Como resultado de la etapa de búsqueda, se obtuvieron 40 artículos, finalmente 11 artículos pasaron al proceso de análisis; donde se identificaron que los algoritmos de optimización más empleado fueron: el que se basa en la Simulación de Montecarlo (27\%) y Programación Lineal (18\%). El 64\% de las investigaciones arrancan el proceso de optimización, sobre la implementación de alguna estrategia de mantenimiento sea preventiva y/o correctiva. Conclusión. Los procesos de optimización buscan minimizar los costos de mantenimiento, maximizar la fiabilidad, minimizar el deterioro del activo o una combinación de ellos.

Palabras claves: Transformador de potencia, optimización del mantenimiento, algoritmo de optimización, costo de mantenimiento, fiabilidad.

\section{Introducción.}

En el Ecuador se han evidenciado cambios en el sector energético en los últimos 12 años por las inversiones realizadas y nueva infraestructura instalada. El parque de generación de energía eléctrica consta de 317 centrales eléctricas que proveen una potencia total efectiva de $8.183 \mathrm{MW}$, (Ministerio de Energía y Recursos Renovables, 2017). Hay ocho nuevas centrales hidráulicas que aportan el $35 \%$ de la generación de potencia; fueron construidas y entraron en operación en los últimos 12 años. Este incremento en la generación le ha permitido al Ecuador cambiar su estatus de importador y convertirse en exportador de energía, (Anchundia Santana, Balderramo Vélez, \& Pico Mera, 2018); su influencia en los ámbitos económico, social y ambiental hacen que sea catalogado como un sector estratégico (Ecuador, 2019). Para lograr tales resultados el estado ecuatoriano invirtió 12.000 millones de dólares en este sector, dinero que se empleó para la construcción y mantenimiento 
de la infraestructura instalada en todas las áreas funcionales que rigen su operación: generación, transmisión, distribución y comercialización (el usuario final) (Anchundia Santana et al., 2018).

Del conjunto de equipos que conforman la infraestructura eléctrica, el transformador de potencia es de los equipos más costosos e importantes, (Murugan \& Ramasamy, 2019), (Nariswari \& Pudjihastuti, 2021); porque es el mecanismo de interconexión entre las etapas de transmisión y distribución. El sistema de distribución cuenta con 324.776 transformadores de voltaje, (MERNNR, 2016), lo que significa que existe una alta inversión en estos equipos. De hecho, el plan "Plan Maestro de Electricidad Expansión y mejora de la distribución 2016-2025”, estableció como objetivo: "Fortalecer los sistemas de distribución para asegurar la calidad y confiabilidad de manera que se incremente la satisfacción del cliente”, (MERNNR, 2016). Es importante señalar que, un fallo en un transformador genera la pérdida inmediata de energía eléctrica en el tramo al que suministra, afecta directamente a la fiabilidad de la red (Murugan \& Ramasamy, 2015), y acarrea pérdidas para el usuario final. Por ejemplo, en Estados Unidos entre el 1997 y 2001 se registraron pérdidas de 286 millones de dólares, causado por fallos en los transformadores, (Liang \& Parlikad, 2018).

Realizar un análisis para conocer los fallos en los transformadores, los modos y sus causas, constituye el primer aspecto en el reto de minimizar la ocurrencia de fallos, porque es la base para el cálculo de la fiabilidad. Los eventos de fallo de un transformador, pueden presentarse en cualquier momento y en cualquiera de sus componentes. En (Madavan \& Balaraman, 2016), se identifica al devanado y núcleo (parte activa del transformador), el cambiador de tomas bajo carga, el buje, el aislamiento y el tanque como los componentes más críticos; debido a su funcionamiento prolongado y al sometimiento continuo a diversas tensiones operativas, provocando su degradación y finalmente el cese de su función.

Según R. Murugan y R. Ramasamy, la reparación de estos equipos es costosa y el tiempo de reemplazo podría tomar hasta un año, por lo cual es importante evitar la ocurrencia de los fallos (Murugan \& Ramasamy, 2019). Para ello y de forma general, hay dos tipos de mantenimiento que se aplican a un equipo si es reparable: mantenimiento preventivo y correctivo. La norma (AENOR EN 13306:2018, 2018), explica que el mantenimiento preventivo tiene el propósitode mitigar la degradación y reducir la probabilidad de fallo. Además (Lee \& Cha, 2016), indica que una buena planificación de mantenimiento preventivo permite mejorar la fiabilidad del equipo. De acuerdo a ( W ang, 2012), aplicar mantenimiento preventivo es una estrategia que contribuye a evitar la ocurrenciade fallos. Este mantenimiento debe ejecutarse antes que ocurra el fallo, y de manera periódica conforme a un cronograma preestablecido o basado en el monitoreo de la condición del equipo.Por otra parte, el mantenimiento correctivo tiene como objeto devolverle al equipo su estado de funcionalidad y debe ser aplicado tan pronto como aparezca el fallo. Para la conservación de un equipo, es necesario realizar actividades combinadas de 
mantenimiento preventivo y correctivo.

El mantenimiento y la fiabilidad están interrelacionados. La fiabilidad es un indicador de mantenimiento que está en función del número de fallos ocurridos en un intervalo de tiempo; puede entenderse como la probabilidad de que un equipo que opera bajo condiciones definidas, cumplasu función requerida sin fallar en un tiempo establecido, (AENOR EN 13306:2018, 2018). En (Bressi, Santos, \& Losa, 2021), se afirma que para lograr un alto nivel de fiabilidad, es decir un valor igual o superior al $95 \%$, la inversión necesaria para mantenimiento se incrementa de manera exponencial, ya que necesariamente se requieren realizar inspecciones más frecuentes. Entonces, es de esperarse que actuar antes de que un equipo entre en estado de avería incrementará su fiabilidad, la pregunta es: ¿cuándo actuar? En efecto, el problema en la elaboración de un plan de mantenimiento radica en definir acertadamente la frecuencia de ejecución de las actividades. En la mayoría de los casos, la forma más común para establecertal frecuencia es hacerlo de forma empírica, estableciendo frecuencias fijas, sin tomar en cuenta que la ocurrencia de los fallos disminuye o se incrementa dependiendo en qué etapa del ciclo devida se encuentre el equipo (Nariswari \& Pudjihastuti, 2021). Otra manera para establecer la frecuencia, es acogerse a las re-comendaciones del fabricante (Murugan \& Ramasamy, 2019), lo cual no considera el contexto operacional del equipo. Esto genera dos escenarios: realizar más intervenciones de mantenimiento preventivo que las requeridas o sencillamente esperar que el fallo ocurra para actuar. En el primer caso, es cierto que se incrementa la fiabilidad del equipo, pero el costo de mantenimiento se encarece excesivamente porel hecho emplearse más recursos (mano de obra, materiales y herramientas). En el segundo caso, actuar correctivamente reduce la vida útil del equipo y genera pérdidas inestimables asociadas a la indisponibilidad del equipo. Si bien, la aplicación de la estrategia de mantenimiento preventivo bien planificado es prometedora, la optimización de su frecuencia de aplicación lo es aún más, yaque contribuirá a la reducción de los costos de mantenimiento; esto es factible mediante métodos de optimización estocástica, dado que el tiempo de ocurrencia de un fallo es un evento aleatorio. Según, (Alaswad \& Xiang, 2017), "La optimización del mantenimiento cumple un rol importante en la efectividad de la operación de cualquier sistema”.

Aunque el diseño de planes preventivos de mantenimiento es una práctica conocida para la industria local, el tema de su optimización es todavía una tarea pendiente. Internacionalmente ya se ha investigado sobre el tema de mantenimiento desde una perspectiva de optimización, enfocándose en ámbitos como: inventario de repuestos y mantenimiento planificado (Wang, 2012), mantenimiento de activos ferroviarios (Bressi et al., 2021), mantenimiento de parques eólicos, (Carlos, Sánchez, Martorell, \& Marton, 2013) y parques eólicos marinos (Irawan, Eskandarpour, Ouelhadj, \& Jones, 2021).

Los modelos de optimización consideran tres aspectos generales: la función objetivo, las variables de decisión y las restricciones (Sánchez, Ramos, Ferrer, Barquín, \& 
Linares, 2010). Según, (Alaswad \& Xiang, 2017), algunos modelos de optimización del mantenimiento basado en la condición, se fundamentan en la maximización de la confiabilidad, disponibilidad o en criterios multiobjetivo (el costo, la disponibilidad, la confiabilidad, etc.).

El problema de optimización multiobjetivo puede ser reducido a un uno de objetivo único, empleando el concepto de efectividad, (Carlos et al., 2013). En (Ramos A., Alonso-Ayuso A., 2011), se explica que en optimización existen problemas lineales estocásticos, donde algunos de los parámetros que intervienen en el modelo son inciertos, por lo cual se consideran como variables aleatorias que pueden ser descritas probabilísticamente a través de distribuciones de probabilidad. En el caso particular del fenómeno de ocurrencia de fallos según (AENOR EN 61649:2012, 2012), (Carlos et al., 2013) y (Nariswari \& Pudjihastuti, 2021), estos eventos pueden modelarse mediante una distribución de Weibull. De hecho, (Nariswari \& Pudjihastuti, 2021), mencionaque "Weibull es la distribución más utilizada y la más beneficiosa para modelar la confiabilidad de un componente o sistema", debido a que la fiabilidad disminuye con el tiempo y los patrones de fallo son diferentes a lo largo de la vida útil de un equipo. Una vez conocidos los parámetros de forma y escala de esta distribución es posible calcular la fiabilidad, probabilidad de fallo y la tasade ocurrencia de fallos del equipo en estudio.

El objetivo de la investigación es identificar los algoritmos de optimización del mantenimiento que sean aplicables a transformadores de potencia, a través de un estudio del estado del arte.

\section{Metodologia}

La revisión de la literatura que se llevó a cabo, se basó en una revisión sistemática de acuerdo a las recomendaciones de (Vera, 2017) y (Karki \& Porras, 2021), con el objetivo de identificar aquellos estudios que portan a identificar las metodologías de optimización del mantenimiento. La metodología empleada consistió en tres etapas generales, la Figura 1. ilustra el procedimiento:

- Etapa 1: Búsqueda de artículos de interés

- Etapa 2: Selección de artículos relevantes

- Etapa 3: Análisis de información seleccionada 


\section{Figura 1}

Procedimiento de selección de artículos

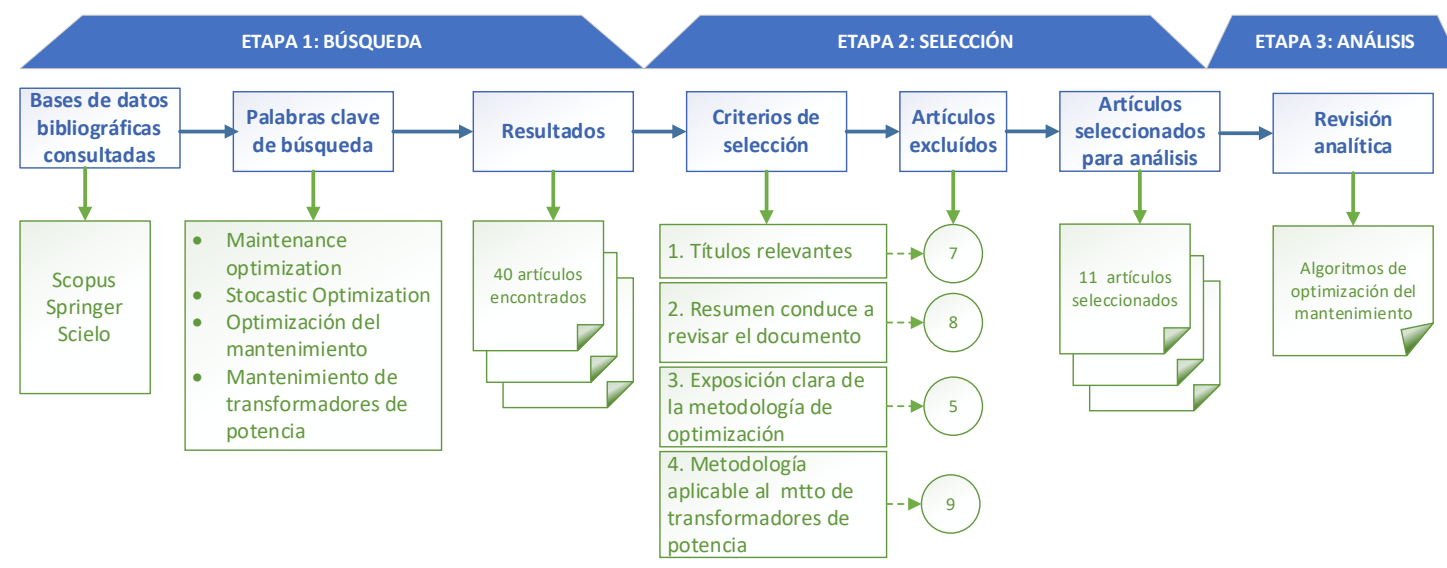

Nota: Descripción de las etapas ejecutadas para la selección de artículos de interés.

Fuente: Los autores

Etapa 1: Búsqueda de artículos de interés

a) Selección de las bases de datos bibliográficas y búsqueda con palabras clave

En correspondencia con el objetivo del presente trabajo, se inició una búsqueda en español en la base de datos Scielo, empleando para la búsqueda la palabra clave “optimización del mantenimiento" y "mantenimiento de transformadores" en donde se obtuvo información limitada, especialmente en el ámbito de la optimización del mantenimiento. Luego se recurrió a revistas especializadas en las bases de datos Scopus y Springer de donde se obtuvo mayor cantidad de información respecto a dos temas específicos, empleando como términos de búsqueda: "maintenance optimization" y "stocastic optimization". Como resultado de la búsqueda se obtuvieron 40 artículos científicos.

Etapa 2: Selección de artículos relevantes

a) Proceso de selección

En esta etapa fue necesario definir bajo qué parámetros se procederá a la selección de información relevante, para obtener como resultado artículos excluidos, aquellos que no cumplen con los criterios de selección y artículos seleccionados, que continuarán a la siguiente etapa.

\section{b) Criterios de selección}

Los criterios básicos de selección, bajos los cuales se filtró la información fueron cuatro:

1. Título relevante

2. Resumen conduce a revisar el documento

3. Exposición clara de la metodología de optimización. 
4. La metodología es aplicable al mantenimiento de transformadores de potencia.

En la primera subetapa, siete artículos no cumplieron con este criterio y fueron rechazados; con los artículos restantes se procedió a valorar con el segundo criterio que consistió en leer detenidamente el resumen, en busca de identificar que sea mencionada la metodología empleada para la optimización del mantenimiento, en este punto se excluyeron siete documentos. En la subetapa tres, se procedió a leer la metodología descrita buscando evidenciar que el algoritmo de optimización sea expuesto con detalle. En incumplimiento de los criterios de selección 29 artículos fueron rechazados y 11 pasaron a la etapa de análisis.

Etapa 3: Análisis de información seleccionada

Una vez identificados aquellos artículos que contribuyen a lograr el objetivo de este trabajo, se analizaron las metodologías descritas, en los cuales se identificó que, para lograr la optimización, cada estudio parte de una estrategia base (la cual se pretende optimizar), para luego usar un algoritmo de optimización; y en efecto el procedimiento de la revisión se esquematizó en la Figura 2, en donde en primera instancia la meta fue identificar la estrategia primaria que cada estudio implementó y luego localizar el algoritmo de optimización.

Figura 2

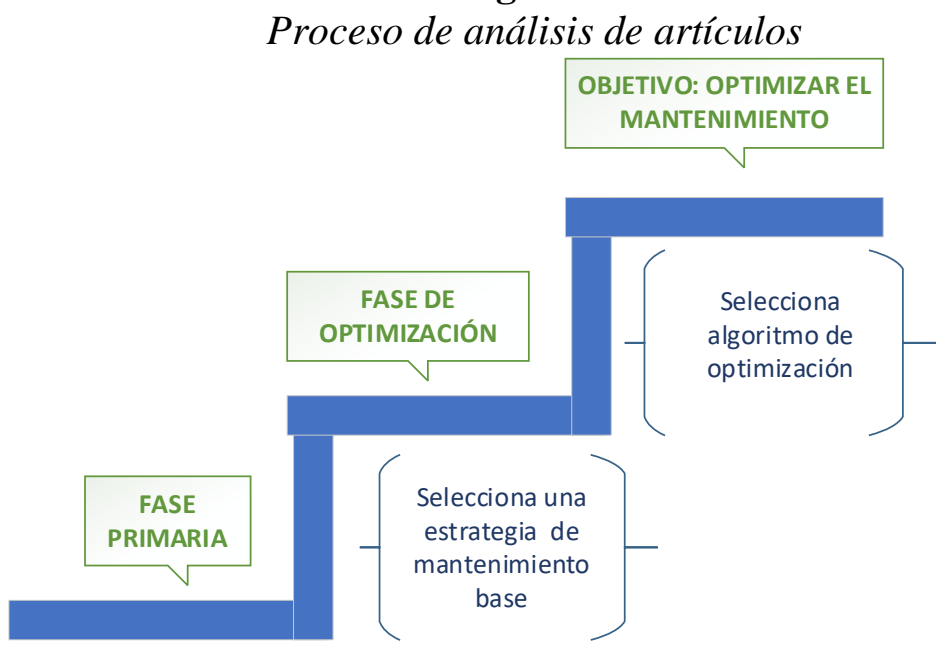

Nota: En las investigaciones analizadas, se identificó que había implementa alguna estrategia de mantenimiento, previo a la optimización.

Fuente: Los autores

\section{Resultados}

Como resultado de las etapas de búsqueda y selección se obtuvieron 11 (de 40) artículos, información que se considera confiable dado que los artículos están publicados en bases de datos científicas reconocidas. Los años de publicación varían desde 2012 y 2021, el $27.2 \%$ de los artículos fueron publicados en el transcurso de este año. En la Figura 3. se presenta una distribución del año de publicación de los artículos clasificados. 


\section{Figura 3}

\section{Distribución de artículos seleccionados y año de publicación}

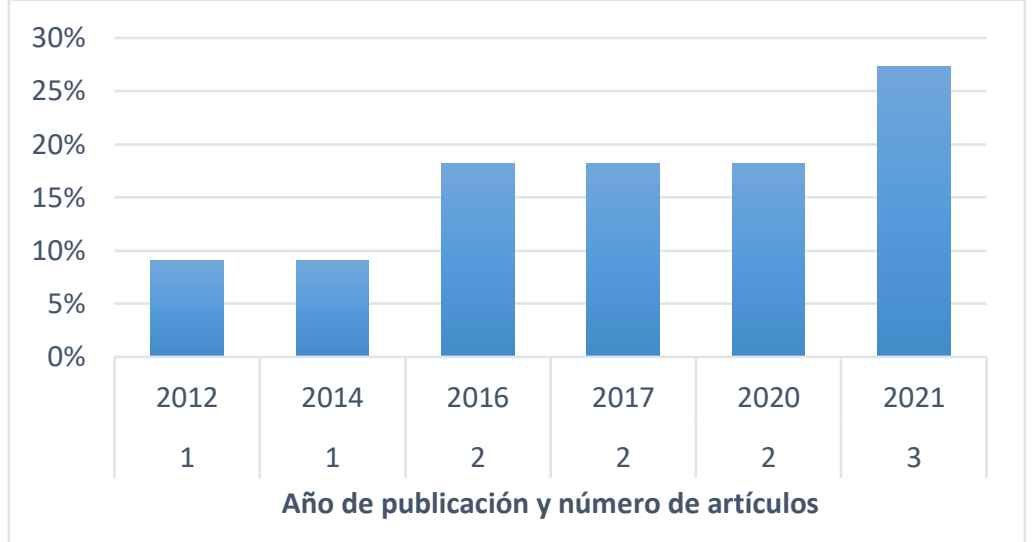

Nota: Progresión de número de artículos publicados referentes a la optimización del mantenimiento en los últimos nueve años.

Fuente: Los autores

Del análisis de los artículos se identificó que en la fase primaria el 64\% de los estudios aplican como estrategia base a optimizar el mantenimiento preventivo y/o correctivo; el 9\% se enfocan en la aplicación de métodos que evalúan la degradación del activo (Coeficiente de reducción de degradación u cadena de Markov); el 9\% aplicó un modelo probabilístico para predicción de fallos a través del cálculo de la fiabilidad, empleando la Distribución de Weibull; el 18\% aplicó otros métodos (ver Figura 4.).

\section{Figura 4}

Métodos o estrategias aplicadas en la fase primaria

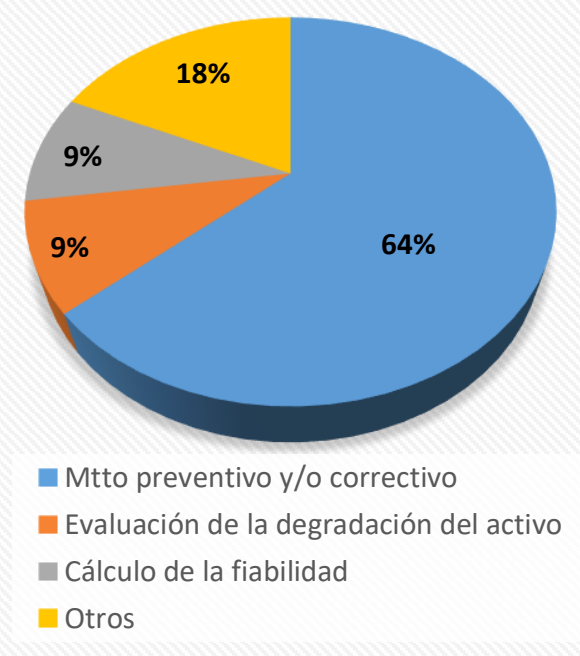

Nota: La estrategia primaria mayormente implementada por las investigaciones analizadas fueron, mantenimiento preventivo, correctivo o una combinación de ellas.

Fuente: Los autores

Del proceso de análisis de la información seleccionada, se obtuvo el modelo o estrategia primaria implementada y se identificó el algoritmo de optimización; así 
como la fuente a la que corresponde la respectiva información (ver Tabla 1). La información se organizó respecto al año de publicación, de manera ascendente.

Tabla 1

Síntesis del análisis de los artículos seleccionados

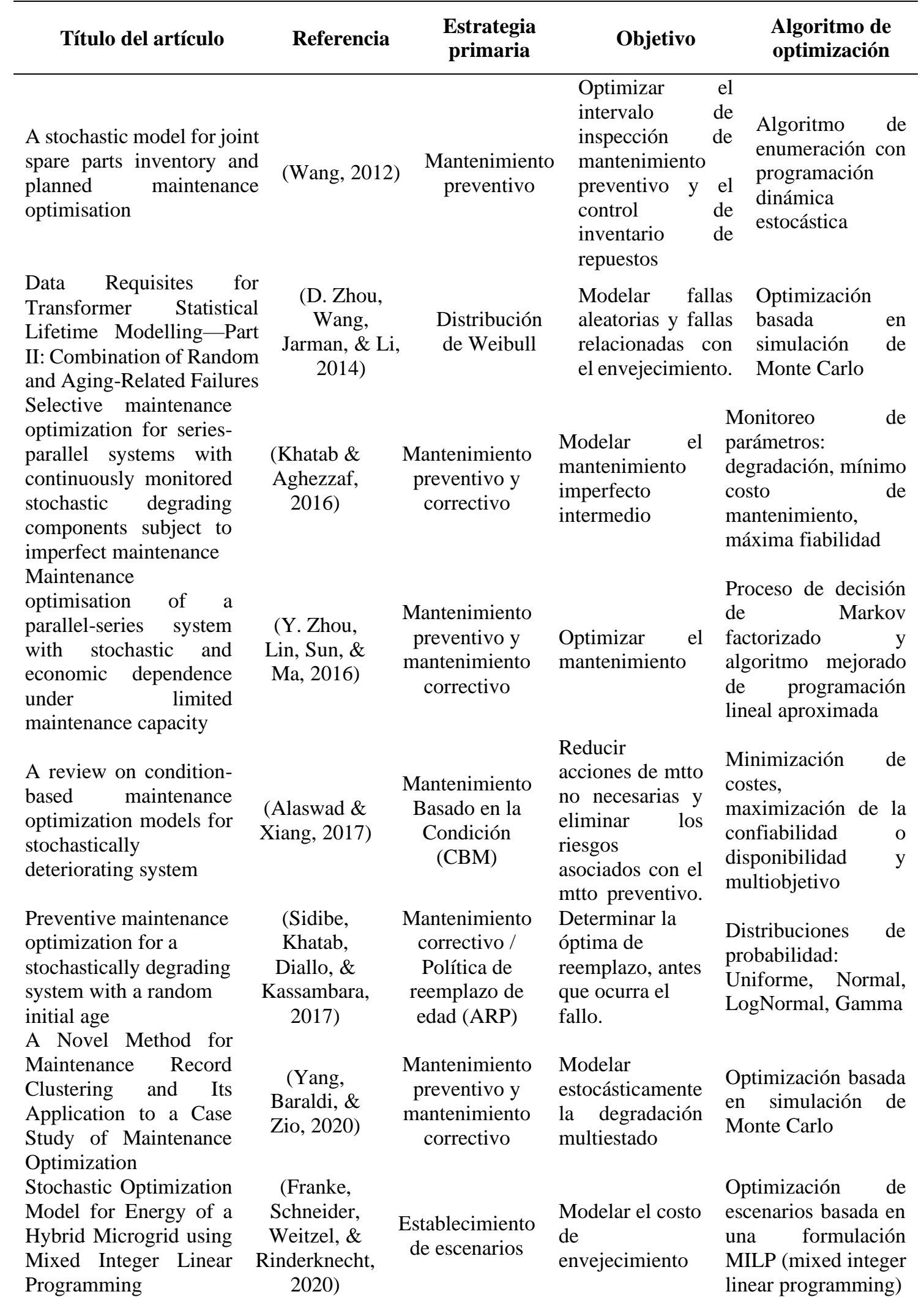




\section{Tabla 1}

Síntesis del análisis de los artículos seleccionados (continuación)

\begin{tabular}{|c|c|c|c|c|}
\hline Título del artículo & Referencia & $\begin{array}{l}\text { Estrategia } \\
\text { primaria }\end{array}$ & Objetivo & $\begin{array}{l}\text { Algoritmo } \\
\text { optimización }\end{array}$ \\
\hline $\begin{array}{l}\text { Simulation-based } \\
\text { optimisation for } \\
\text { stochastic maintenance } \\
\text { routing in an offshore } \\
\text { wind farm }\end{array}$ & $\begin{array}{c}\text { (Irawan et al., } \\
\text { 2021) }\end{array}$ & $\begin{array}{c}\text { Metaheurística } \\
\text { de búsqueda de } \\
\text { grades } \\
\text { vecindarios. }\end{array}$ & $\begin{array}{lr}\text { Enrutar el } & \text { mtto } \\
\text { para } & \text { un } \\
\text { horizonte } & \text { de } \\
\text { planificación } & \text { de } \\
\text { un día. } & \end{array}$ & $\begin{array}{l}\text { Optimización basada } \\
\text { en simulación de } \\
\text { Monte Carlo }\end{array}$ \\
\hline $\begin{array}{lr}\text { Optimization } & \text { of } \\
\text { maintenance } & \text { strategies } \\
\text { for railway } & \text { track-bed } \\
\text { considering } & \\
\text { probabilistic } & \\
\text { degradation models and } \\
\text { different } & \text { reliability } \\
\text { levels } & \end{array}$ & $\begin{array}{l}\text { (Bressi et al., } \\
\text { 2021) }\end{array}$ & $\begin{array}{l}\text { Cadena de } \\
\text { Markov }\end{array}$ & $\begin{array}{ll}\text { Predecir } & \text { la } \\
\text { degradación } & \text { del } \\
\text { lecho de la vía } \\
\text { del tren. }\end{array}$ & $\begin{array}{l}\text { Algoritmo genético } \\
(\mathrm{GA})\end{array}$ \\
\hline $\begin{array}{l}\text { A cost-effective two- } \\
\text { stage optimization } \\
\text { model for microgrid } \\
\text { planning and scheduling } \\
\text { with compressed air } \\
\text { energy storage and } \\
\text { preventive maintenance }\end{array}$ & $\begin{array}{l}\text { (Gao et al., } \\
\text { 2021) }\end{array}$ & $\begin{array}{l}\text { Mantenimiento } \\
\text { preventivo y } \\
\text { mantenimiento } \\
\text { correctivo }\end{array}$ & $\begin{array}{l}\text { Planificar el } \\
\text { mantenimiento }\end{array}$ & $\begin{array}{l}\text { Estrategia evolutiva } \\
\text { de depredadores y } \\
\text { presas basada en } \\
\text { múltiples presas } \\
\text { (MPEPPS). }\end{array}$ \\
\hline
\end{tabular}

Fuente: Los autores

La Figura 5, indica los algoritmos que los artículos seleccionados emplearon, observándose que los dos algoritmos más empleados con el propósito de optimizar el mantenimiento fueron Simulación de Montecarlo y Programación Lineal. En la Figura 6, se esquematiza de manera general las estrategias o métodos que emplearon los artículos tanto en la fase primaria como en la fase de optimización.

\section{Figura 5}

Algoritmos de optimización del mantenimiento

Algoritmo genético (GA)

Nota: Algoritmos más empleados por las investigaciones analizadas para optimizar el mantenimiento. Fuente: Los autores 


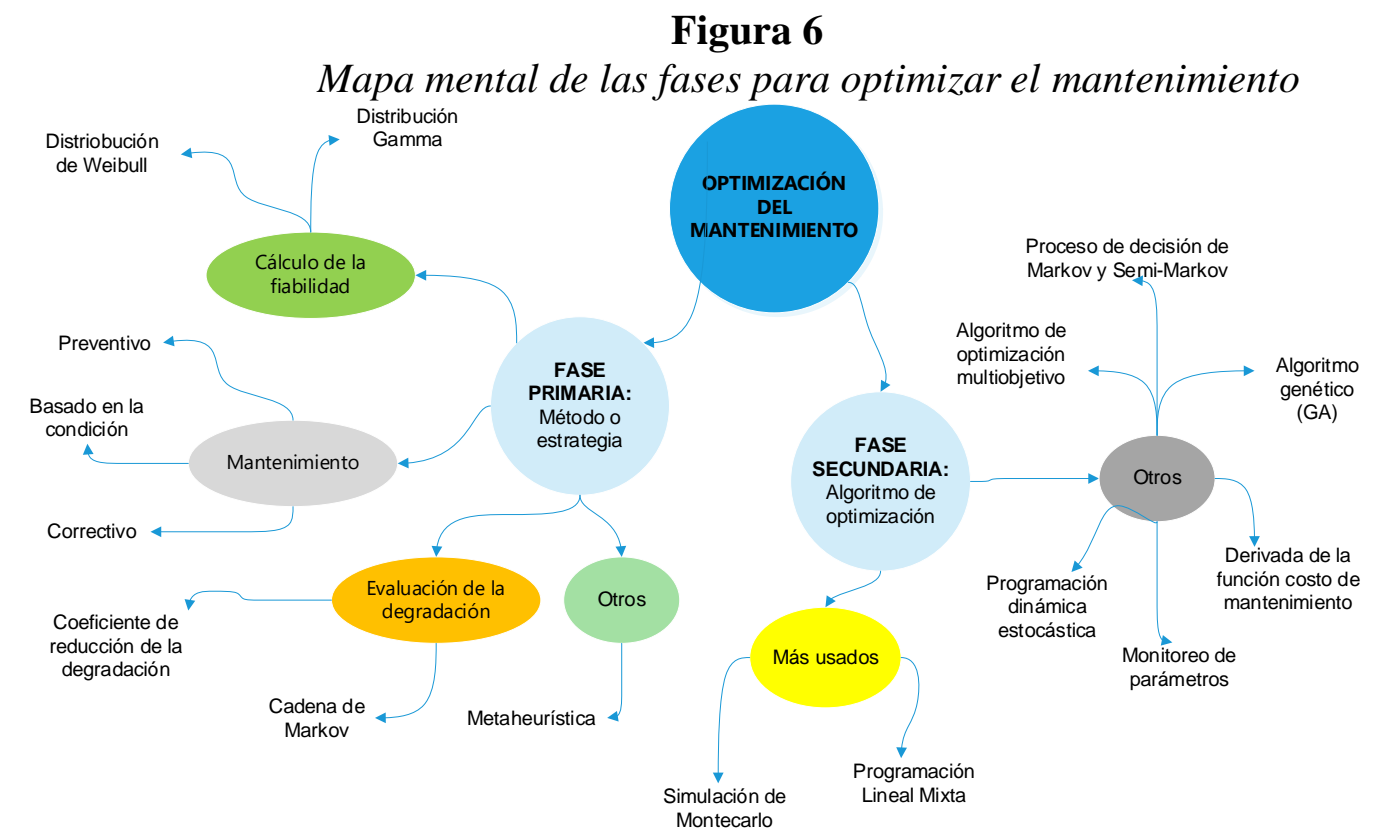

Nota: Síntesis de las estrategias primarias y metodologías de optimización, empleadas en las 11 investigaciones analizadas.

Fuente: Los autores

\section{Discusión}

La optimización del mantenimiento en países desarrollados es una estrategia que ya se está aplicado, pues se evidenció que evitar la ocurrencia de fallos sin estimar los costos asociados resulta inadecuado y obsoleto. El impacto positivo de la optimización del mantenimiento sobre la vida de los activos, radica en permitir que estos cumplan su función de manera efectiva y más productiva. En general este enfoque moderno contribuye a que las industrias sean más competitivas, y perciban réditos económicos, pues la optimización del mantenimiento se enfoca en minimizar el costo del mantenimiento preventivo y/o correctivo, en algunos casos maximizando la fiabilidad y monitoreando la degradación del activo.

\section{Conclusiones}

El análisis de los artículos seleccionados, permitió concluir que:

- Todos los estudios de optimización del mantenimiento, tienen implementado inicialmente un programa que será optimizado, sea de mantenimiento preventivo, basado en la condición o correctivo; análisis de fiabilidad, o evaluación de la degradación del activo.

- Los algoritmos más usados fueron el algoritmo de optimización basado en simulación de Montecarlo y Programación Lineal.

- En la mayoría de los casos la optimización del mantenimiento se basó en la minimización del costo de mantenimiento combinado con la maximización de la fiabilidad. 


\section{Referencias bibliográficas:}

AENOR EN 13306:2018. Terminología del mantenimiento. , AeUNE. (2018). Terminología del mantenimiento. Aenor, 31. https://www.une.org/encuentra-tunorma/busca-tu-norma/norma/?c=N0026303nor $\S(2018)$.

AENOR EN 61649:2012. Análisis de Weibull. , (2012).

Alaswad, S., \& Xiang, Y. (2017). A review on condition-based maintenance optimization models for stochastically deteriorating system. Reliability Engineering and System Safety, 157, 54-63. https://doi.org/10.1016/j.ress.2016.08.009

Anchundia Santana, P. E., Balderramo Vélez, N. R., \& Pico Mera, G. E. (2018). Causas y Efectos que Impulsaron la Innovación del Sector Eléctrico Ecuatoriano. Revista de Investigaciones en Energía, Medio Ambiente y Tecnología: RIEMAT ISSN: 25880721, 3(2), 18. https://doi.org/10.33936/riemat.v3i2.1626

Bressi, S., Santos, J., \& Losa, M. (2021). Optimization of maintenance strategies for railway track-bed considering probabilistic degradation models and different reliability levels. Reliability Engineering and System Safety, 207(October 2020). https://doi.org/10.1016/j.ress.2020.107359

Carlos, S., Sánchez, A., Martorell, S., \& Marton, I. (2013). Onshore wind farms maintenance optimization using a stochastic model. Mathematical and Computer Modelling, 57(7-8), 1884-1890. https://doi.org/10.1016/j.mcm.2011.12.025

Ecuador, C. Del. (2019). Normas Constitucionales - Sectores Estratégicos. Ministero De Telecuminicaciones Y De Las Sociedad De Informacion, 53(9), 1689-1699.

Franke, G., Schneider, M., Weitzel, T., \& Rinderknecht, S. (2020). Stochastic Optimization Model for Energy Management of a Hybrid Microgrid using Mixed Integer Linear Programming. IFAC-PapersOnLine, 53(2), 12948-12955. https://doi.org/10.1016/j.ifacol.2020.12.2132

Gao, J., Chen, J. J., Qi, B. X., Zhao, Y. L., Peng, K., \& Zhang, X. H. (2021). A costeffective two-stage optimization model for microgrid planning and scheduling with compressed air energy storage and preventive maintenance. International Journal of Electrical Power and Energy Systems, 125(October 2020). https://doi.org/10.1016/j.ijepes.2020.106547

Irawan, C. A., Eskandarpour, M., Ouelhadj, D., \& Jones, D. (2021). Simulation-based optimisation for stochastic maintenance routing in an offshore wind farm. European Journal of Operational Research, 289(3), 912-926. https://doi.org/10.1016/j.ejor.2019.08.032

Karki, B. R., \& Porras, J. (2021). Digitalization for sustainable maintenance services: A systematic literature review. Digital Business, 1(2), 100011. https://doi.org/10.1016/j.digbus.2021.100011 
Khatab, A., \& Aghezzaf, E. H. (2016). Selective maintenance optimization for seriesparallel systems with continuously monitored stochastic degrading components subject to imperfect maintenance. IFAC-PapersOnLine, 49(28), 256-261. https://doi.org/10.1016/j.ifacol.2016.11.044

Lee, H., \& Cha, J. H. (2016). New stochastic models for preventive maintenance and maintenance optimization. European Journal of Operational Research, 255(1), 8090. https://doi.org/10.1016/j.ejor.2016.04.020

Liang, Z., \& Parlikad, A. (2018). A Markovian model for power transformer maintenance. International Journal of Electrical Power and Energy Systems, 99(September 2017), 175-182. https://doi.org/10.1016/j.ijepes.2017.12.024

Madavan, R., \& Balaraman, S. (2016). Failure analysis of transformer liquid - solid insulation system under selective environmental conditions using Weibull statistics method. Engineering Failure Analysis, 65, 26-38. https://doi.org/10.1016/j.engfailanal.2016.03.017

MERNNR. (2016). Plan Maestro de Electricidad Expansión y mejora de la distribución 2016-2025. 239-273. Recuperado de https://www.recursosyenergia.gob.ec/planmaestro-de-electricidad/

Ministerio de Energía y Recursos Renovables. (2017). Plan Maestro de la Electricidad 2016-2025. Expansión de la generación. Recuperado de https://www.recursosyenergia.gob.ec/wp-content/uploads/2020/01/4.EXPANSION-DE-LA-GENERACION.pdf

Murugan, R., \& Ramasamy, R. (2015). Failure analysis of power transformer for effective maintenance planning in electric utilities. Engineering Failure Analysis, 55, 182192. https://doi.org/10.1016/j.engfailanal.2015.06.002

Murugan, R., \& Ramasamy, R. (2019). Understanding the power transformer component failures for health index-based maintenance planning in electric utilities. Engineering Failure Analysis, 96(July 2018), 274-288. https://doi.org/10.1016/j.engfailanal.2018.10.011

Nariswari, R., \& Pudjihastuti, H. (2021). Reliability Analysis of Distribution Transformer with Bayesian Mixture and Cox Regression Approach. Procedia Computer Science, 179(2020), 305-312. https://doi.org/10.1016/j.procs.2021.01.010

Ramos A., Alonso-Ayuso A., P. G. (2011). Optimización bajo incertidumbre (Universi d).

Sánchez, P., Ramos, A., Ferrer, J., Barquín, J., \& Linares, P. (2010). Modelos matemáticos de optimización. Universidad Pontificia Comillas, 55.

Sidibe, I. B., Khatab, A., Diallo, C., \& Kassambara, A. (2017). Preventive maintenance optimization for a stochastically degrading system with a random initial age. 
Reliability Engineering and System Safety, 159(November 2016), 255-263. https://doi.org/10.1016/j.ress.2016.11.018

Vera, O. (2017). Artículos de Revisión. Cómo escribir artículos de revisión. Revista Chilena de Ortopedia y Traumatología, 58(02), 033-033. https://doi.org/10.1055/s0037-1606585

Wang, W. (2012). A stochastic model for joint spare parts inventory and planned maintenance optimisation. European Journal of Operational Research, 216(1), 127139. https://doi.org/10.1016/j.ejor.2011.07.031

Yang, Z., Baraldi, P., \& Zio, E. (2020). A novel method for maintenance record clustering and its application to a case study of maintenance optimization. Reliability $\begin{array}{lllll}\text { Engineering } \quad \text { and System } & \text { Safety, }\end{array}$ https://doi.org/10.1016/j.ress.2020.107103

Zhou, D., Wang, Z., Jarman, P., \& Li, C. (2014). Data requisites for transformer statistical lifetime modelling - Part II: Combination of random and aging-related failures. IEEE Transactions on Power Delivery, 29(1), 154-160. https://doi.org/10.1109/TPWRD.2013.2270116

Zhou, Y., Lin, T. R., Sun, Y., \& Ma, L. (2016). Maintenance optimisation of a parallelseries system with stochastic and economic dependence under limited maintenance capacity. Reliability Engineering \& System Safety, 155, 137-146. https://doi.org/10.1016/J.RESS.2016.06.012

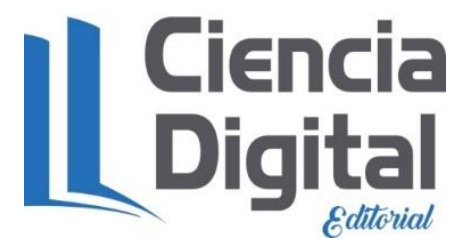




\section{PARA CITAR EL ARTÍCULO INDEXADO.}

Villacrés-Parra, S. R., Viscaíno-Cuzco, M. A., \& Gallegos-Londoño, C. M. (2021). Metodologías de optimización del mantenimiento enfocado a transformadores de potencia: Una revisión del estado del arte. ConcienciaDigital, 4(3.1), 238-252. https://doi.org/10.33262/concienciadigital.v4i3.1.1827

\section{Liencia}

El artículo que se publica es de exclusiva responsabilidad de los autores y no necesariamente reflejan el pensamiento de la Revista Conciencia Digital.

El artículo queda en propiedad de la revista y, por tanto, su publicación parcial y/o total en otro medio tiene que ser autorizado por el director de la Revista Conciencia Digital.

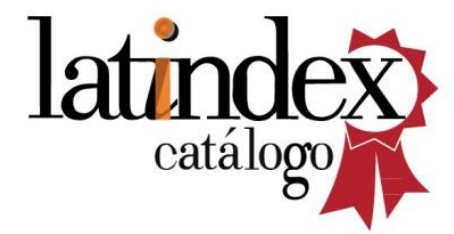

